\title{
Kullback-Leibler Divergence Approach to Partitioned Update Kalman Filter
}

\author{
Matti Raitoharju, Ángel F. García-Fernández, and Robert Piché
}

\begin{abstract}
Kalman filtering is a widely used framework for Bayesian estimation. The partitioned update Kalman filter applies a Kalman filter update in parts so that the most linear parts of measurements are applied first. In this paper, we generalize partitioned update Kalman filter, which requires the use oft the second order extended Kalman filter, so that it can be used with any Kalman filter extension. To do so, we use a Kullback-Leibler divergence approach to measure the nonlinearity of the measurement, which is theoretically more sound than the nonlinearity measure used in the original partitioned update Kalman filter. Results show that the use of the proposed partitioned update filter improves the estimation accuracy.
\end{abstract}

Index Terms-Bayesian estimation; nonlinear; estimation; Kalman filters; Kullback-Leibler divergence

\section{INTRODUCTION}

In Bayesian filtering we are interested in calculating the probability density function (PDF) of a dynamic state based on a sequence of measurements. It is a recursive process in which a prior distribution is updated using a measurement to obtain a posterior distribution. This distribution then evolves in time to become a new prior distribution. Bayesian estimation has a wide range of applications from positioning [1], tracking [2], and quality control [3], to brain imaging [4] and modeling spread of infectious diseases [5]. In general, a Bayesian estimate cannot be computed in closed form. Under certain conditions, which include that measurements and state transition function have to be linear and associated noises Gaussian, Bayesian estimates can be computed in closed form using the algorithm known as the [6]. For nonlinear measurements various Kalman filter extensions have been developed. When the nonlinearity is small, the Kalman filter (KF) extensions produce accurate estimates, but large nonlinearity can cause serious inaccuracies.

Several methods have been proposed to quantify the amount of nonlinearity in order to monitor the performance of a KF extension [7]-[13]. An algorithm based on second order Extended Kalman filter (EKF2) was presented in [7, p. 349] and it was extended for multidimensional correlated measurements in [8]. Although it was found to be a good indicator of the accuracy of a KF extension compared to methods that are computationally feasible presented in [9]-[11], its evaluation requires computation of second order derivatives of the measurement function or computation of the measurement function values in a number of points that increases quadratically.

The effect of the nonlinearity can also be gauged by comparing the higher order moments of the posterior to those corresponding to the Gaussian assumption. This is considered for example in [12].
If the prior covariance is sufficiently small, any nonlinear function is well approximated as a linear function in the prior's high-probability region. Therefore, if a nonlinearity measure indicates that a measurement is highly nonlinear, the prior can be split into a sum of small-covariance parts that can be used as the components in a Gaussian mixture filter (GMF) [14]. As the computational burden increases with every split, the number of new components needed to bring the nonlinearity measure below a certain threshold should be minimized [15].

In [16] another use of the EKF2-based nonlinearity measure in [8] was presented. The algorithm, called the partitioned update Kalman filter (PUKF), transforms a measurement vector with a linear transformation so that the nonlinearity of the least nonlinear measurement element is minimized. Only the elements with nonlinearity below a threshold are applied first and then the linearization and evaluation of the nonlinearity is redone. This way the estimates become more accurate.

In [13], it was shown that the moments computed in a general Gaussian filter (GGF) [17] can be used to compute the Kullback-Leibler divergence (KLD) [18] of the GGF approximation to the joint PDF of the state and the measurement from the true joint PDF. Various KF extensions can be seen as approximations of the GGF and, thus, they can be used to approximate this KLD, which can be also interpreted as a nonlinearity measure. This KLD has been used in Kalman optimization in [19].

In this paper, we present an algorithm that is similar to PUKF, but uses the KLD based nonlinearity measure. The benefits of using the KLD measure is that it is mathematically sound and it can be used in combination with any GGF approximation that can be used to approximate the KLD. If the proposed algorithm is used with EKF2 or a numerical approximation of it, the results are the same as with the original PUKF.

The rest of this paper is organized as follows: Section II gives the background work. The new algorithm is developed in Section III. Section IV] presents examples of the use and accuracy of the proposed algorithm. Section $\mathrm{V}$ concludes the paper.

\section{BACKGROUND WORK}

In this paper, we derive an improved version of the PUKF. To do so, it is convenient to introduce some background material. In Section II-A, we first present the GGF update and a measure of its performance based on the KLD. In Section II-B, we revisit the PUKF. 


\section{A. $G G F$}

In this paper, we consider the Bayesian update step of a state. We assume that state $x \in \mathbb{R}^{n}$ has a Gaussian prior PDF

$$
p(x)=\mathrm{N}\left(x \mid \mu^{-}, P^{-}\right) .
$$

where $\mathrm{N}$ is the PDF of a normal distribution, $\mu^{-}$is the prior mean, and $P^{-}$is the prior covariance. This state is observed through a measurement that is modeled with a measurement model of form

$$
y=h(x)+\varepsilon,
$$

where $y$ is the $d$-dimensional measurement value and $\varepsilon$ is a zero-mean Gaussian measurement noise with covariance $R$.

The objective is to compute the posterior

$$
p(x \mid y) \propto p(x) p(y \mid x),
$$

where $\propto$ stands for proportionality and $p(y \mid x)=\mathrm{N}(y \mid h(x), R)$ is the density of the measurement given the state. If function $h(\cdot)$ is linear, the posterior can be computed exactly using the $\mathrm{KF}$. But when $h(\cdot)$ is not linear, an approximation has to be used.

A general way to formulate a Kalman filter type approximation is to use the GGF formulation for additive noise [17]. In the update step, we approximate the posterior as a Gaussian with mean $\mu^{+}$and covariance $P^{+}$by

$$
\begin{aligned}
& \mu^{+}=\mu^{-}+K(y-\hat{y}) \\
& P^{+}=P^{-}-K S K^{T},
\end{aligned}
$$

where

$$
\begin{aligned}
\hat{y} & =\int h(x) p(x) \mathrm{d} x \\
\Psi & =\int\left(x-\mu^{-}\right)(h(x)-\hat{y})^{T} p(x) \mathrm{d} x \\
\Phi & =\int(h(x)-\hat{y})(h(x)-\hat{y})^{T} p(x) \mathrm{d} x \\
S & =\Phi+R \\
K & =\Psi S^{-1} .
\end{aligned}
$$

The GGF is implicitly defining a Gaussian approximation of the joint density of state $x$ and measurement $y$. The approximation, denoted $q(x, y)$ is not the exact joint density $p(x, y)$. The approximation error can be measured using KLD [18]

$$
\eta=\mathrm{KLD}(p, q)=\iint p(x, y) \log \frac{p(x, y)}{q(x, y)} \mathrm{d} x \mathrm{~d} y
$$

In [13], it was shown that

$$
\eta=\frac{1}{2} \log \left|I+R^{-1} \Upsilon\right|
$$

where

$$
\Upsilon=\Phi-\Psi^{T}\left(P^{-}\right)^{-1} \Psi
$$

Different KF extensions, such as the unscented Kalman filter (UKF) [20] and the cubature Kalman filter (CKF) [21], can be interpreted as approximations of the GGF and they can also be used to compute approximations of the integrals (6)-(8) and, thus, approximate the joint KLD. Some KF extensions, such as the extended Kalman filter (EKF), make linearizations such that the moments (7) and (8) can be written in the form

$$
\begin{aligned}
& \Psi=P^{-} A^{T} \\
& \Phi=A P^{-} A^{T},
\end{aligned}
$$

where $A$ is a $d \times n$ matrix. For these filters $\Upsilon$ in $(13)$ is always 0 and they cannot be used to approximate the KLD.

\section{B. PUKF}

For conditionally independent measurements $y_{1}, \ldots, y_{d}$ given the state we can write

$$
p\left(y_{1: d} \mid x\right)=\prod_{i=1}^{d} p\left(y_{i} \mid x\right) .
$$

The prior can be updated using the measurements sequentially:

$$
\begin{aligned}
p\left(x \mid y_{1: n}\right) & \propto p(x) p\left(y_{1} \mid x\right) \prod_{i=2}^{d} p\left(y_{i} \mid x\right) \\
& \propto p\left(x \mid y_{1}\right) \prod_{i=2}^{d} p\left(y_{i} \mid x\right) .
\end{aligned}
$$

When the measurement model is of the form (2) and the noise covariance $R$ is diagonal, the measurements are conditionally independent. Thus, in a linear Gaussian measurement model, the KF update can be applied one measurement element at a time [22, p. 119] and the posterior distribution does not change. However, when the updates are approximate, the final posterior PDF approximation changes.

In general, the $d$ measurement elements are not conditionally independent given the state so (16) does not hold. The main idea behind the PUKF [16] is to apply a linear transformation to the measurement model so that measurement elements are conditionally independent and the measurements are applied in an order that aims to minimize the approximation error. A general description of PUKF is given in Algorithm 1

The PUKF in [16] can only be used with EKF2 [7, pp. 345-347], which is based on second order Taylor expansion of the measurement function, or with a central difference filter [17] that is a numerical approximation of EKF2. We call this numerical approximation the numerical second order Extended

\section{Algorithm 1: A general description of the PUKF algo- rithm}

1 Evaluate the nonlinearity of the measurement elements.

2 Minimize the nonlinearity of part of the measurement by applying a linear transformation to the measurement.

3 Update the state using the part of the measurement whose nonlinearity is smaller than a set threshold.

4 If the whole measurement is not applied, use the partially updated prior as a new prior and the unused measurements as a new measurement and return to 1 .

5 Return posterior. 

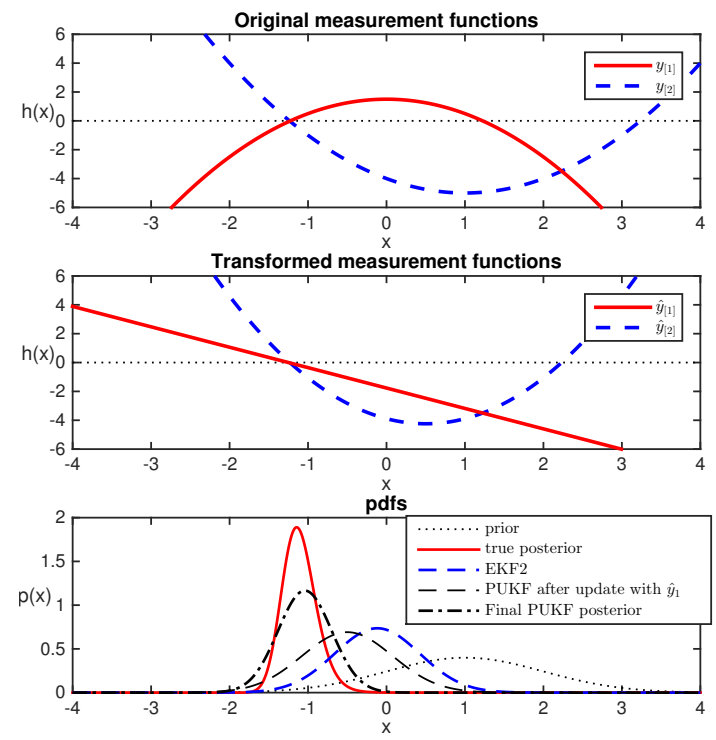

Fig. 1. Transforming second order polynomial measurements to minimize nonlinearity of $\hat{y}_{1}$ and posterior comparison of PUKF and EKF2

Kalman filter (nEKF2). The EKF2-specific nonlinearity measure is

$$
\hat{\eta}=\sum_{i=1}^{d} \sum_{j=1}^{d}\left(R^{-1}\right)_{[i, j]} \operatorname{tr} P H_{i} P H_{j},
$$

where $H_{i}$ is the Hessian of $i$ th element of the measurement function $h(\cdot)$ or its numerical approximation and subscript $[i, j]$ means the matrix element in the $i$ th row and $j$ th column.

Example 1: We proceed to illustrate how the PUKF works as it is the foundation of the methods we propose in this paper. We consider an example from [16]. Here the prior is one dimensional with $\operatorname{PDF} \mathrm{N}(x \mid 1,1)$ and the nonlinear measurement equation is

$$
y=\left[\begin{array}{c}
x^{2}-2 x-4 \\
-x^{2}+\frac{3}{2}
\end{array}\right]+\varepsilon,
$$

where $\varepsilon$ has PDF $\mathrm{N}(\varepsilon \mid 0, I)$. The measurement can be transformed with the linear transformation

$$
\hat{y}=\frac{1}{\sqrt{2}}\left[\begin{array}{cc}
1 & 1 \\
1 & -1
\end{array}\right] y
$$

into a linear term and a polynomial term:

$$
\hat{y}=\sqrt{2}\left[\begin{array}{c}
-x-\frac{5}{4} \\
x^{2}-x-\frac{11}{4}
\end{array}\right]+\hat{\varepsilon},
$$

where $\hat{\varepsilon} \sim \mathrm{N}(0, I)$. In PUKF the linear measurement function is applied first and the partially updated state has mean $-\frac{1}{2}$ and covariance $\frac{1}{3}$. The polynomial measurement function is applied using this partially updated state and EKF2-based update. In Figure 1 EKF2 is used as a reference. EKF2 applies both measurements at once and the posterior estimate is the same for the original and transformed measurement models. When compared to the true posterior, which is computed using a dense grid, the posterior estimate of PUKF is significantly more accurate than the EKF2 posterior estimate.

\section{KULlBACK-LEIBLER PARTITIONED UPDATE KALMAN FILTER (KLPUKF)}

In this paper we develop KLPUKF that uses (12) as the nonlinearity measure. Thus, algorithm is not limited to EKF2 based filters and any filter, such as UKF [20] or CKF [21], that is a good enough approximation of the GGF can be used. As noted earlier, some algorithms, such as EKF, produce always 0 nonlinearity. Thus, they are not good enough approximations of the GGF. We assume that the KF extension does the moment approximations at the prior. Iterative Kalman filter extensions, such as iterated extended Kalman filter (IEKF) [7. pp. 349-351] and iterated posterior linearization filter (IPLF) [23], are not considered in this paper to be used with the KLPUKF.

We recall that the steps of the PUKF algorithm are given in Algorithm 1. Therefore, in this section we specify how to compute the nonlinearity and the linear transformation. The linear transformation of the measurement model can be written as

$$
\tilde{y}=D y=D h(x)+D \varepsilon=\tilde{h}(x)+\tilde{\varepsilon},
$$

where $D$ is a nonsingular square matrix. In the appendix we show that the linear transformation 22 does not change the posterior if all measurements are applied at once (Proof A) and does not have an effect on the nonlinearity measure 12 (Proof A.

However, if an update is split into a sequence of independent measurement updates, the posterior may change. We use a transformation such that the transformed noise covariance $\tilde{R}$ is

$$
\tilde{R}=\operatorname{cov} \tilde{\varepsilon}=D R D^{T}=I .
$$

We show in Proof $\mathrm{A}$ in the Appendix that the nonlinearity (12) can be written now as

$$
\eta=\frac{1}{2} \log |I+\tilde{\Upsilon}|
$$

where

$$
\tilde{\Upsilon}=D \Upsilon D^{T}
$$

We further want $\tilde{\Upsilon}$ to be diagonal. Now the nonlinearity measure of the $i$ th measurement element can be defined as

$$
\tilde{\eta}_{i}=\frac{1}{2} \log \left(1+\tilde{\Upsilon}_{[i, i]}\right) \text {. }
$$

When $\tilde{R}=I$ and $\tilde{\Upsilon}$ is diagonal the nonlinearity of the least nonlinear measurement element is minimized. This is shown in Proof $\mathrm{A}$ in the Appendix. Thus, this gives the best choice for a single element update.

Rather than choosing $\tilde{R}=I$, we could have chosen any other diagonal matrix. However, this would increase the value of the corresponding diagonal values of $\tilde{\Upsilon}$ and the KLD values of individual measurements of (53) would not change. Thus, choosing $\tilde{R}=I$ is an arbitrary, but natural choice.

To compute the matrix $D$ we first introduce the notation for matrix square root

$$
\sqrt{R} \sqrt{R}^{T}=R
$$


which can be computed with, e.g., Cholesky decomposition. The transformation $D$ that makes $\tilde{\Upsilon}$ diagonal and $\tilde{R}=I$ is [16]

$$
D=U^{T} \sqrt{R}^{-1},
$$

where $U$ is computed using an eigendecomposition

$$
U \Lambda U^{T}=\sqrt{R}^{-1} \Upsilon \sqrt{R}^{-T},
$$

where $U$ is orthogonal and the eigenvalues in the diagonal matrix $\Lambda$ are sorted in ascending order. We can see that this transformation fulfills our requirements of having identity transformed measurement noise covariance 23 from

$$
\tilde{R}=U^{T} \sqrt{R}^{-1} R \sqrt{R}^{-T} U=I
$$

and the diagonality of matrix $\tilde{R}$ (25) can be seen from orthogonality and 29]

$$
\tilde{\Upsilon}=U^{T} \sqrt{R}^{-1} \Upsilon \sqrt{R}^{-T} U=\Lambda .
$$

To save computational resources, rather than using only one measurement in each update, we perform the update using all measurement elements that have $\tilde{\eta}_{i}$ below a limit $\eta_{\text {limit }}$ or if this set is empty then with the measurement element with smallest $\tilde{\eta}_{i}$. We also note that after a measurement update the moments (6)-(8) are recomputed. Because the nonlinearities of the measurements change, the transformation matrix $D$ is recomputed between every update.

The KLPUKF algorithm is given in Algorithm 2 When using EKF2 or a numerical approximation of it as basis of the filter and setting $\eta_{\text {limit }}$ properly, the algorithm produces the same results as with the original PUKF.

\section{Simulation EXAMPles}

In this section, we present examples of how the KLPUKF improves estimation accuracy. First, we evaluate the accuracy enhancements for a one-dimensional state that is observed through measurements containing trigonometric functions. Then we present examples with range measurements to beacons. In these examples we also test filtering and evaluate the effect of the nonlinearity limit $\eta_{\text {limit. }}$ In our examples, we use an approximation of GGF computed in a dense grid, UKF [20], nEKF2 [17], and smart sampling Kalman filter ( $\left.\mathrm{S}^{2} \mathrm{KF}\right)$ [24].

Example 2: 1D example

First we consider an example with a 1-dimensional state and standard normal prior. The prior is updated with a 3-element measurement of form

$$
y=\left[\begin{array}{c}
x+4 \sin (x)+7 \\
-x+4 \sin (x)-4 \\
-2 \cos (x)-8
\end{array}\right]+\varepsilon,
$$

where PDF of $\varepsilon$ is $\mathrm{N}(\varepsilon \mid 0, I)$. The top-left plot of Figure 2 shows these measurement functions. The dashed lines show the statistical linearizations of the functions obtained from moments (6)-(8) [23]. The dashed black line is the prior mean and dotted lines show the $2 \sigma$ limits; i.e. $95 \%$ of the probability is within these limits. The numbers in the legend are the KLDs (53) of each element of the measurement function computed

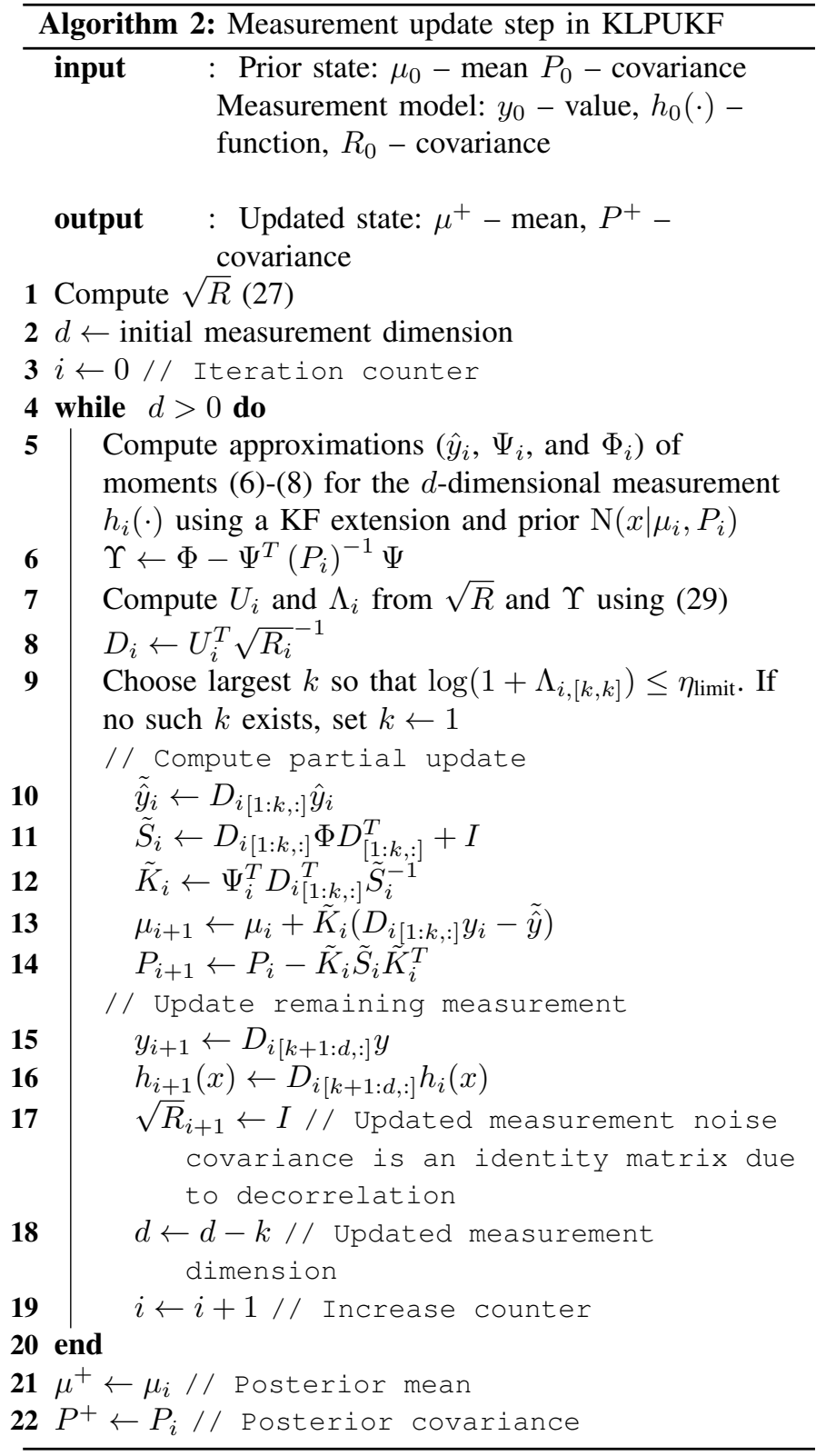

using (53). Because $\Upsilon$ is not diagonal for initial functions, their KLDs does not sum to the total KLD that is 0.8533 for the initial measurements. The sum of KLDs of transformed functions is the same as the total KLD.

Before transformation, the function with the cosine term (blue) has the smallest KLD. For these measurements and prior the transformation matrix is

$$
D=\left[\begin{array}{ccc}
-\frac{1}{\sqrt{2}} & \frac{1}{\sqrt{2}} & 0 \\
0 & 0 & 1 \\
-\frac{1}{\sqrt{2}} & -\frac{1}{\sqrt{2}} & 0
\end{array}\right],
$$

which causes the sin terms in the new first component to cancel out and the new measurements are

$$
y=\left[\begin{array}{c}
-\sqrt{2} x-\frac{11}{\sqrt{2}} \\
-2 \cos x-4 \\
-4 \sqrt{2} \sin (x) \frac{3}{\sqrt{2}}
\end{array}\right]+\varepsilon .
$$



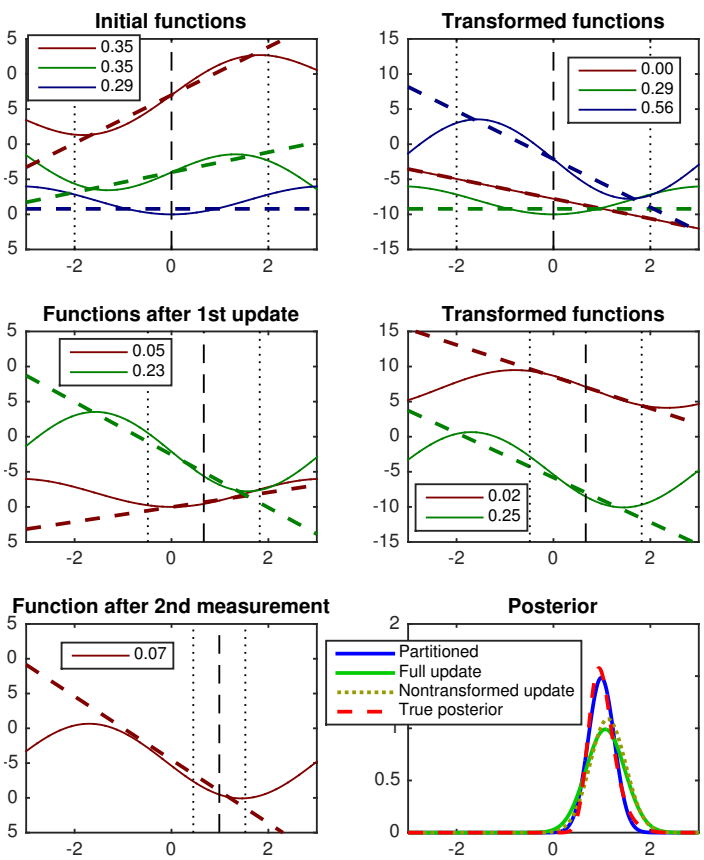

Fig. 2. Example updates using all measurements at once and using KLPUKF Red corresponds to $y_{1}$, green to $y_{2}$, and blue to $y_{3}$.

The top-right plot shows these transformed measurements and corresponding KLDs. The linear measurement naturally has a zero KLD and is applied first. The second row shows the remaining components of the transformed functions and the new linearizations that are made in the new prior. The last row shows the last measurement and the posterior PDFs. The curve labeled "partitioned" is the posterior computed with the proposed algorithm. "Full update" is the update using all measurements simultaneously and "nontransformed update" is the update made in parts without applying transformation $D$ i.e. the measurement with the cosine element is applied first because it has the smallest KLD. This plot shows that the posterior computed with the proposed method is the most accurate one.

Example 3: Range measurements

In this example, we consider a more realistic situation with 2-dimensional state with prior PDF $\mathrm{N}(x \mid \mathbf{0}, 12 I)$ updated with 3 range measurements. The measurement model is

$$
h(x)=\left[\begin{array}{l}
\sqrt{\left(x_{1}-2\right)^{2}+\left(x_{2}-2\right)^{2}} \\
\sqrt{\left(x_{1}+6\right)^{2}+\left(x_{2}-6\right)^{2}} \\
\sqrt{\left(x_{1}+2\right)^{2}+\left(x_{2}-1\right)^{2}}
\end{array}\right]+\varepsilon,
$$

where $\varepsilon$ has $\operatorname{PDF} \mathrm{N}(\varepsilon \mid 0, I)$ and the measurement values are $\left[\begin{array}{lll}5 & 11.5 & 3.5\end{array}\right]^{T}$.

Figure 3 shows an example of the update with KLPUKF. The top row in Figure 3 shows the likelihoods of the range measurements (blue), prior (red) and the measurement likelihoods when linearized using the GGF (orange). The second row shows the transformed measurements and their linearizations within the original prior. The last row shows the linearizations within the prior and how the prior and linearizations change after partial updates. The integrals for GGF were computed using a dense grid.

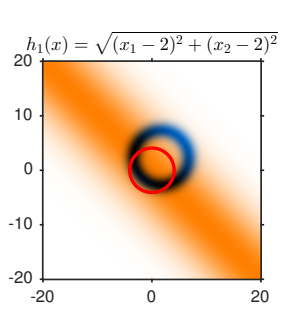

Original measurements
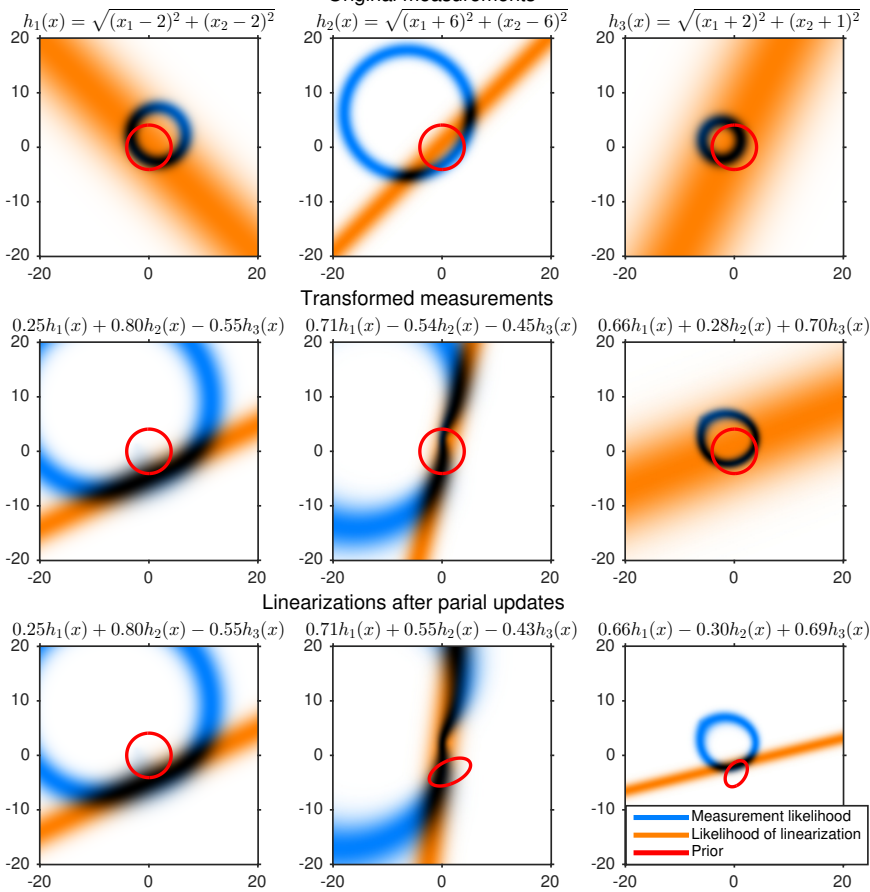

Fig. 3. Example updates with using all measurements at once and with using KLPUKF with GGF. Ellipses contain $50 \%$ of the prior probability. Intensity of the color of the likelihoods represents its value.

We can see that the third measurement of both nontransformed and transformed measurements has a large uncertainty when linearized using the original prior. When using the partially updated prior the third measurement has much smaller uncertainty.

Figure 4 shows the same example computed using the UKF linearizations. Contours containing $50 \%$ of the posterior probability computed using GGF, UKF, nEKF2 and their KLPUKF counterparts are shown in Figure 5. The contour for true posterior is computed in a grid. Figure shows how the GGF produces a larger covariance than the true covariance and how the use of KLPUKF makes the estimate closer to the true estimate. The UKF estimate has a bad shape: it is too narrow horizontally and too long vertically. Using KLPUKF with UKF reduces the covariance in the too long direction and the resulting posterior estimate is closer to the true posterior.

\section{Example 4: Filtering example}

In the filtering example we consider the same measurement model as in the previous example. We use a 4-dimensional state

$$
x=\left[\begin{array}{llll}
r_{1} & r_{2} & v_{1} & v_{2}
\end{array}\right]^{T},
$$

where $r_{1}$ and $r_{2}$ are position variables and $v_{1}$ and $v_{2}$ velocity variables. The state transition model is

$$
x_{t+1}=F x_{t}+\varepsilon_{Q},
$$



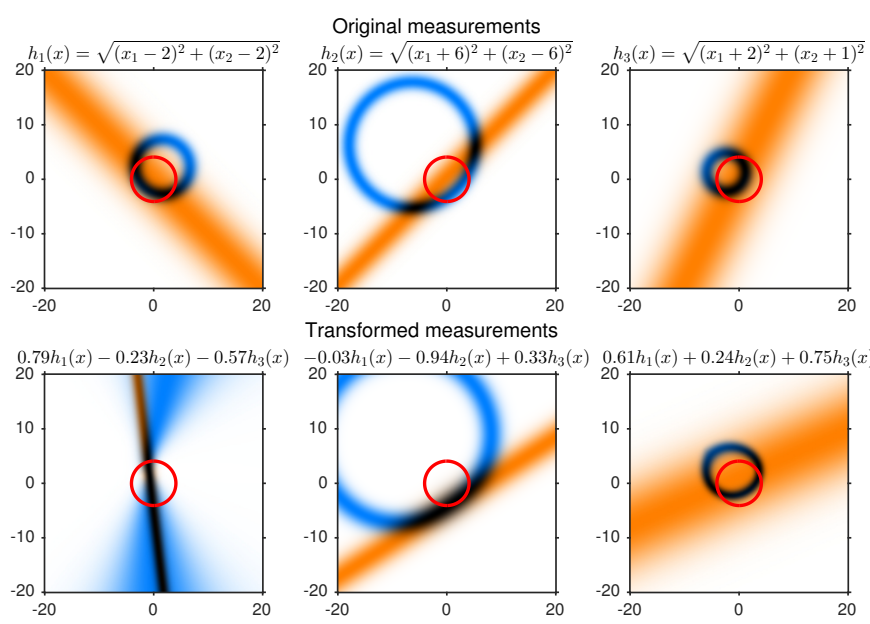

Transformed measurements
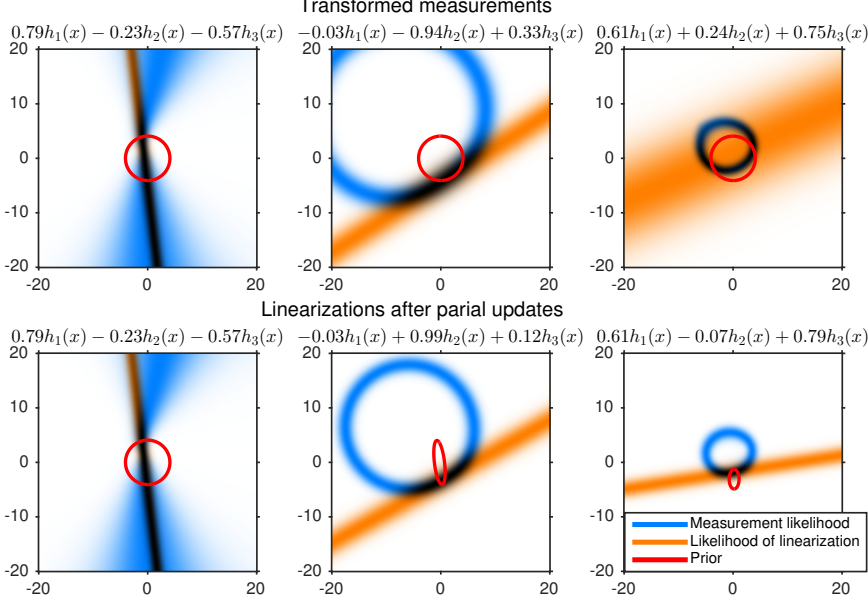

Fig. 4. Example updates with using all measurements at once and with using KLPUKF and UKF. Ellipses contain $50 \%$ of the prior probability. Intensity of the color of the likelihoods represent its value.

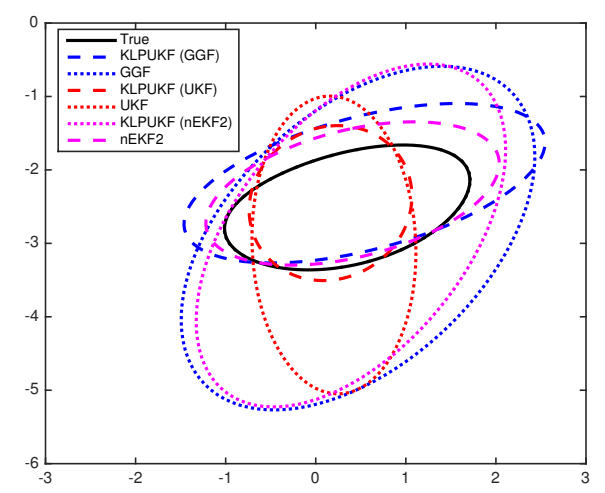

Fig. 5. Contours containing 50\% of the posterior probability obtained with different algorithms

where

$$
\begin{gathered}
F=\left[\begin{array}{llll}
1 & 0 & 1 & 0 \\
0 & 1 & 0 & 1 \\
0 & 0 & 1 & 0 \\
0 & 0 & 0 & 1
\end{array}\right] \\
p\left(\varepsilon_{Q}\right)=\mathrm{N}\left(\varepsilon_{Q} \mid 0,\left[\begin{array}{cccc}
0 & 0 & 0 & 0 \\
0 & 0 & 0 & 0 \\
0 & 0 & 0.04 & 0 \\
0 & 0 & 0 & 0.04
\end{array}\right]\right) .
\end{gathered}
$$

TABLE I

MEAN OF FILTERING ERRORS AT THE FIRST TIME STEP

\begin{tabular}{r|c|cc|cc} 
& All & \multicolumn{2}{|c|}{ Sequential } & \multicolumn{2}{c}{ KLPUKF } \\
EKF & 2.35 & 1.95 & $-16.7 \%$ & & \\
nEKF2 & 1.87 & 1.72 & $-8.0 \%$ & 1.48 & $-20.9 \%$ \\
UKF & 1.87 & 1.69 & $-10.0 \%$ & 1.54 & $-17.9 \%$ \\
S $^{2}$ KF & 1.82 & 1.69 & $-7.5 \%$ & 1.46 & $-20.0 \%$
\end{tabular}

TABLE II

MEAN OF FILTERING ERRORS AT THE LAST TIME STEP

\begin{tabular}{r|c|cc|cc} 
& All & \multicolumn{2}{|c|}{ Sequential } & \multicolumn{2}{c}{ KLPUKF } \\
\hline EKF & 2.17 & 1.99 & $-8.1 \%$ & & \\
nEKF2 & 1.59 & 1.64 & $3.1 \%$ & 1.50 & $-5.6 \%$ \\
UKF & 1.67 & 1.70 & $1.5 \%$ & 1.57 & $-6.2 \%$ \\
$S^{2}$ KF & 1.59 & 1.62 & $1.5 \%$ & 1.49 & $-6.5 \%$
\end{tabular}

The prior is normal with covariance

$$
P_{0}=\left[\begin{array}{cccc}
12 & 0 & 0 & 0 \\
0 & 12 & 0 & 0 \\
0 & 0 & 1 & 0 \\
0 & 0 & 0 & 1
\end{array}\right] .
$$

To have variation in the initial linearizations we sample the initial mean $\mu_{0}$ from the normal distribution that has a zero mean and covariance $P_{0}$. In this test we used Algorithm 2 with KLD threshold $\eta_{\text {limit }}=0$, so that each measurement is processed separately at every time step. We tested the KLPUKF with moments computed using nEKF2, UKF and $S^{2} \mathrm{KF}$ [24]. $S^{2} \mathrm{KF}$ allows to select the number of sigmapoints. We used in our tests 64 sigma-points for $\mathrm{S}^{2} \mathrm{KF}$ while the UKF used 9 sigma-points and nEKF2 used 16 sigmapoints. The nonlinearity threshold is set to $\eta_{\text {limit }}=0$ so each measurement element is applied separately. We also tested how the estimation accuracy changes if the measurement elements are applied sequentially in a random order. Estimates are also computed with EKF. Because EKF produces always a zero nonlinearity the KLPUKF cannot improve the result from the applying the measurements in a random order. The routes were simulated 10000 times.

Table I shows the mean of errors after the first update. The "all" column uses all measurements at once, the "sequential" column uses the measurements in random order and the third column uses KLPUKF. The table shows how the use of KLPUKF instead of the standard filter improves the estimate accuracy more than $15 \%$ at the first time step. Also we can see that the application of the measurements sequentially improves the estimation accuracy.

Table [I] shows the results of the last time step of the routes. In these results we can see that the sequential application of measurements improves only the estimation accuracy of EKF; other filters have worse accuracy, but the KLPUKF improves the accuracy. The smaller improvement gained by the KLPUKF can be explained with the observation that the route moved usually outside the sources of the range measurements and the measurement geometry is worse i.e. there is less information in the actual measurements, while they are more linear than in the first step.

This example showed that the use of KLPUKF gives accuracy improvements and that the use of independent measurements sequentially does not necessarily improve the accuracy. 


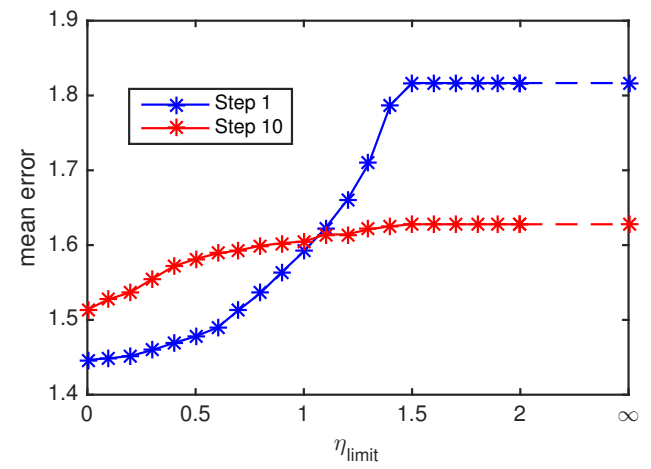

Fig. 6. Mean errors as a function of $\eta_{\text {limit }}$

To get an insight of how to choose $\eta_{\text {limit }}$ we evaluated the previous example with the $S^{2} \mathrm{KF}$, which we assume to be the best approximation of the GGF of used filters, with different values for $\eta_{\text {limit }}$. Values were set to range from 0 to 2 with interval 0.1 and with $\eta_{\text {limit }}=\infty$, which corresponds to the standard $\mathrm{S}^{2} \mathrm{KF}$.

Figure 6 shows how the mean error varies with $\eta_{\text {limit }}$. First of all we can see that in this test setup all measurements had $\eta<1.5$ so having $\eta_{\text {limit }} \geq 1.5$ does not change the outcome at all. At the last time step the saturation point of error is already achieved at $\eta_{\text {limit }} \geq 1.5$. At the first and last time step having $\eta_{\text {limit }}=0.2$ would not increase the error much. At the beginning of the track $\eta_{\text {limit }}=0.5$ has only a small effect compared to having $\eta_{\text {limit }}=0.5$ at the end of the track. This behavior may be caused by the accumulation of the errors during the track.

\section{CONCLUSIONS}

We have presented KLPUKF, an algorithm to perform Bayesian updates with nonlinear multi-dimensional measurements. The key idea is to perform the update in several steps such that the measurement elements with lower nonlinearity are processed first. The measurement elements' processing order is selected so that the joint KLD of the state and the measurement is minimized.

The proposed algorithm can be used with GGF approximations, such as sigma-point filters, to improve the estimation accuracy as the nonlinearity measure (KLD) can be computed using variables that are already computed within the filter. Simulation results have demonstrated better accuracy of KLPUKF compared to several existing filters.

\section{ACKNOWLEDGEMENT}

M. Raitoharju works in OpenKin project that is funded by the Academy of Finland.

\section{REFERENCES}

[1] P. Müller, H. Wymeersch, and R. Piché, "UWB positioning with generalized Gaussian mixture filters," IEEE Transactions on Mobile Computing, vol. 13, no. 10, pp. 2406-2414, Oct 2014.

[2] S. Sadhu, S. Mondal, M. Srinivasan, and T. Ghoshal, "Sigma point Kalman filter for bearing only tracking," Signal Processing, Special Section: Multimodal Human-Computer Interfaces, vol. 86, no. 12, pp. 3769 - 3777, 2006, doi 10.1016/j.sigpro.2006.03.006
[3] M. Zhai and S. Fu, "Applying target maneuver onset detection algorithms to defects detection in aluminum foil," Signal Processing, vol. 90, no. 7, pp. 2319 - 2326, 2010, doi 10.1016/j.sigpro.2010.02.012

[4] P. Hiltunen, S. Särkkä, I. Nissilä, A. Lajunen, and J. Lampinen, "State space regularization in the nonstationary inverse problem for diffuse optical tomography," Inverse Problems, vol. 27, no. 2, p. 025009, 2011. [Online]. Available: http://stacks.iop.org/0266-5611/27/i=2/a=025009

[5] M. J. Keeling and P. Rohani, Modeling infectious diseases in humans and animals. Princeton: Princeton University Press, 2008. [Online]. Available: http://opac.inria.fr/record=b1124487

[6] Y. Ho and R. Lee, "A Bayesian approach to problems in stochastic estimation and control," IEEE Transactions on Automatic Control, vol. 9, no. 4, pp. 333 - 339, October 1964, doi 10.1109/TAC.1964.1105763

[7] A. H. Jazwinski, Stochastic Processes and Filtering Theory, ser. Mathematics in Science and Engineering. Academic Press, 1970, vol. 64.

[8] M. Raitoharju, "Linear models and approximations in personal positioning," Ph.D. dissertation, Tampere University of Technology, November 2014. [Online]. Available: http://URN.fi/URN:ISBN: 978-952-15-3421-8

[9] F. Faubel, J. McDonough, and D. Klakow, "The split and merge unscented Gaussian mixture filter," IEEE Signal Processing Letters, vol. 16, no. 9, pp. 786 -789, September 2009, doi $10.1109 /$ LSP.2009.2024859

[10] F. Havlak and M. Campbell, "Discrete and continuous, probabilistic anticipation for autonomous robots in urban environments," IEEE Transactions on Robotics, vol. PP, no. 99, pp. 1-14, 2013, doi $10.1109 /$ TRO.2013.2291620

[11] M. Huber, "Adaptive Gaussian mixture filter based on statistical linearization," in Proceedings of the 14th International Conference on Information Fusion (FUSION), July 2011, pp. 1-8. [Online]. Available: http://ieeexplore.ieee.org/xpl/articleDetails.jsp?arnumber=5977694

[12] O. Straka, J. Dunik, and M. Simandl, "Measures of non-Gaussianity in unscented Kalman filter framework," in Proceedings of the 17th International Conference on Information Fusion (FUSION), July 2014, pp. 1-8. [Online]. Available: http://ieeexplore.ieee.org/xpl/articleDetails. jsp?arnumber $=6916118$

[13] M. Morelande and A. García-Fernández, "Analysis of Kalman filter approximations for nonlinear measurements," IEEE Transactions on Signal Processing, vol. 61, no. 22, pp. 5477-5484, Nov 2013, doi $10.1109 /$ TSP.2013.2279367

[14] H. W. Sorenson and D. L. Alspach, "Recursive Bayesian estimation using Gaussian sums," Automatica, vol. 7, no. 4, pp. 465-479, 1971, doi $10.1016 / 0005-1098(71) 90097-5$

[15] M. Raitoharju, S. Ali-Loytty, and R. Piche, "Binomial Gaussian mixture filter," EURASIP Journal on Advances in Signal Processing, vol. 2015, no. 1 , p. 36, 2015, doi $10.1186 / \mathrm{s} 13634-015-0221-2$

[16] M. Raitoharju, R. Piché, J. Ala-Luhtala, and S. Ali-Löytty, "Partitioned update Kalman filter," ISIF Journal of Advances in Information Fusion, in press. [Online]. Available: http://arxiv.org/abs/1503.02857

[17] K. Ito and K. Xiong, "Gaussian filters for nonlinear filtering problems," IEEE Transactions on Automatic Control, vol. 45, no. 5, pp. 910-927, May 2000, doi $10.1109 / 9.855552$

[18] S. Kullback and R. A. Leibler, "On information and sufficiency," The Annals of Mathematical Statistics, vol. 22, no. 1, pp. 79-86, 1951. [Online]. Available: http://www.jstor.org/stable/2236703

[19] Á. F. García-Fernández and L. Svensson, "Gaussian MAP filtering using Kalman optimization," IEEE Transactions on Automatic Control, vol. 60, no. 5, pp. 1336-1349, May 2015, doi 10.1109/TAC.2014.2372909

[20] E. Wan and R. Van der Merwe, "The unscented Kalman filter for nonlinear estimation," in Proceedings of the Adaptive Systems for Signal Processing, Communications, and Control Symposium. AS-SPCC., 2000, pp. 153-158, doi 10.1109/ASSPCC.2000.882463

[21] I. Arasaratnam and S. Haykin, "Cubature Kalman filters," IEEE Transactions on Automatic Control, vol. 54, no. 6, pp. 1254-1269, June 2009, doi $10.1109 /$ TAC.2009.2019800

[22] P. S. Maybeck, Stochastic models, estimation, and control, ser. Mathematics in Science and Engineering. Academic Press, 1979, vol. 1.

[23] A. García-Fernández, L. Svensson, M. Morelande, and S. Särkka, "Posterior linearization filter: Principles and implementation using sigma points," IEEE Transactions on Signal Processing, vol. 63, no. 20, pp. 5561-5573, Oct 2015, doi 10.1109/TSP.2015.2454485

[24] J. Steinbring and U. D. Hanebeck, "S KF: The smart sampling Kalman filter," in Proceedings of the 16th International Conference of Information Fusion (FUSION). Istanbul: IEEE, 2013, pp. 20892096. [Online]. Available: http://ieeexplore.ieee.org/xpl/articleDetails. jsp?arnumber $=6641263$ 


\section{APPENDIX}

Proof: GGF estimate does not change when a linear transformation is applied to measurements

Substituting (22) to the integrals (6)-(8) the new expectations are

$$
\begin{aligned}
& \tilde{\hat{y}}=\int D h(x) p(x) \mathrm{d} x=D \hat{y} \\
& \tilde{\Psi}=\int\left(x-\mu^{-}\right)(D h(x)-D \hat{y})^{T} p(x) \mathrm{d} x=\Psi D^{T} \\
& \tilde{\Phi}=\int(D h(x)-D \hat{y})(D h(x)-D \hat{y})^{T} p(x) \mathrm{d} x=D \Phi D
\end{aligned}
$$

and the measurement noise covariance of the transformed measurement is

$$
\tilde{R}=D R D^{T} .
$$

Substituting these into (4), (5), (9), and (10)

$$
\begin{aligned}
\tilde{S} & =D(\Phi+R) D^{T} \\
\tilde{K} & =\Psi D^{T} D^{-T} S^{-1} D^{-1}=K D^{-1} \\
\tilde{\mu^{+}} & =\mu^{-}+K D^{-1}(D y-D \hat{y})=\mu^{+} \\
\tilde{P^{+}} & =P^{-}-K D^{-1} D S D^{T} D^{-T} D^{T} K^{T}=P^{+}
\end{aligned}
$$

we see that the posterior computed using the transformed measurement does not change.

Proof: Total KLD nonlinearity does not change under linear transformation

Using (42) and (43) in (13) we get

$$
\begin{aligned}
\tilde{\Upsilon} & =\tilde{\Phi}-\tilde{\Psi}^{T}\left(P^{-}\right)^{-1} \tilde{\Psi} \\
& =D\left(\Phi-\Psi^{T}\left(P^{-}\right)^{-1} \tilde{\Psi}\right) D^{T} \\
& =D \Upsilon D^{T}
\end{aligned}
$$

By substituting this into (12) with transformed measurement noise covariance (44)

$$
\begin{aligned}
\tilde{\eta} & =\frac{1}{2} \log \left|I+\left(D R D^{T}\right)^{-1} D \Upsilon D^{T}\right| \\
& =\frac{1}{2} \log \left|D^{-T} D^{T}+D^{-T} R^{-1} \Upsilon D^{-T}\right| \\
& =\frac{1}{2} \log \left(\left|D^{-T}\right|\left|I+R^{-1} \Upsilon\right|\left|D^{T}\right|\right) \\
& =\frac{1}{2} \log \left|I+R^{-1} \Upsilon\right| \\
& =\eta
\end{aligned}
$$

we see that the total nonlinearity does not change.

Proof: Nonlinearity of an element is minimized

The proof is very similar to one in the Appendix B in [16]. When $\tilde{R}=I$ and $\widetilde{\Upsilon}$ are diagonal the total nonlinearity is

$$
\begin{aligned}
\tilde{\eta} & =\frac{1}{2} \log |I+\tilde{\Upsilon}| \\
& =\sum_{i=1}^{d} \frac{1}{2} \log \left(1+\tilde{\Upsilon}_{[i, i]}\right)
\end{aligned}
$$

and the nonlinearity corresponding to $i$ th measurement element is

$$
\tilde{\eta}_{i}=\frac{1}{2} \log \left(1+\tilde{\Upsilon}_{[i, i]}\right)
$$

We will show that the smallest diagonal element of $\tilde{\Upsilon}$ is as small as possible under a linear transformation that preserves $R=I$ and further that the second smallest diagonal element is as small as possible, when the next smallest is as small as possible etc. If the measurement model is transformed by multiplying it with matrix $V$, the transformed variables are $\hat{\Upsilon}=V \tilde{\Upsilon} V^{T}$ and $\hat{R}=V I V^{T}=V V^{T}$. Because we want to have $R=I, V$ has to be unitary. The $i$ th diagonal element of the transformed matrix $\tilde{\Upsilon}$ is $v_{i}^{T} \tilde{\Upsilon} v_{i}=\sum_{j=1}^{d} v_{i,[j]}^{2} \tilde{\Upsilon}_{[j, j]}$, where $v_{i}$ is the $i$ th column of $V$. Because $V$ is unitary $\sum_{j=1}^{d} v_{i,[j]}^{2}=$ 1 and the $i$ th diagonal element of the transformed matrix $\hat{\Upsilon}$ is

$$
\sum_{j=1}^{d} v_{i,[j]}^{2} \tilde{\Upsilon}_{[j, j]} \geq \sum_{j=1}^{d} v_{i,[j]}^{2} \min _{j}\left\{\tilde{\Upsilon}_{[j, j]}\right\}=\min _{j}\left\{\Upsilon_{[j, j]}\right\}
$$

Thus, the new diagonal element cannot be smaller than the smallest diagonal element of $\tilde{\Upsilon}$.

If the smallest element is the first element of the diagonal the possible transformation for the second smallest element is

$$
\hat{\Upsilon}=\left[\begin{array}{cc}
1 & 0^{T} \\
0 & V
\end{array}\right] \tilde{\Upsilon}\left[\begin{array}{cc}
1 & 0^{T} \\
0 & V^{T}
\end{array}\right]
$$

With the same reasoning as given already the second diagonal has to be already the smallest possible. Inductively this applies to all diagonal elements. One could also show that now the measurement element corresponding to the maximal element of $\tilde{\Upsilon}$ has the largest possible KLD. 